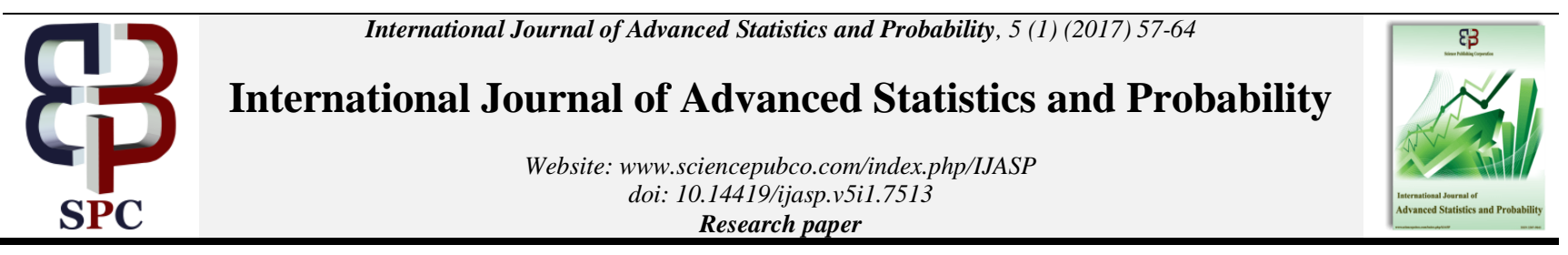

\title{
The beta compound Rayleigh distribution : Properties and applications
}

\author{
Hesham M. Reyad ${ }^{1 *}$, Soha A. Othman ${ }^{2}$ \\ ${ }^{1}$ College of Business and Economics, Qassim University, Kingdom of Saudi Arabia \\ ${ }^{2}$ Institute of Statistical Studies and Research, Cairo University, Egypt \\ *Corresponding author_E-mail: hesham_reyad@yahoo.com
}

\begin{abstract}
In this paper, we introduce a new four parameter continuous model, called the beta compound Rayleigh (BCR) distribution that extends the compound Rayleigh distribution. Basic properties of the proposed distribution such as; mean, variance, coefficient of variation, raw and incomplete moments, skewness, kurtosis, moment and probability generating functions, reliability analysis, Lorenz, Bonferroni and Zenga curves, Rényi of entropy, order statistics and record statistics are investigated. We obtain the maximum likelihood estimates and the observed information matrix for the model parameters. Two real data sets are used to illustrate the usefulness of the new model.
\end{abstract}

Keywords: Beta Distribution; Compound Rayleigh Distribution; Maximum Likelihood Estimation; Order Statistics; Record Statistics.

\section{Introduction}

The compound Rayleigh (CR) distribution plays a vital role for modeling and analysis in different areas of statistics, including reliability study and life time data, especially in biological and medical science. In the last couple of decades, statisticians have much attention to study this distribution. Abushal [1] applied the maximum likelihood and Bayes approaches to estimate parameters, reliability and hazard functions of the $\mathrm{CR}$ distribution based on progressive first-failure censoed data. Shajaee et al. [2] obtained the empirical Bayes estimates for parameter and reliability function associated with the $\mathrm{CR}$ distribution under record data. Barot and Patal [3] compared the maximum likelihood and Bayes estimates of the reliability parameters corresponding to the $\mathrm{CR}$ distribution under progressive type-ii censored data. Abd-Elmougod and Mahmoud [4] studied the CR distribution with constant partially accelerated life tests under an adaptive type-ii propgressive hybrid censored data.

The random variable $\mathrm{X}$ with $\mathrm{CR}$ distribution has cumulative distribution function (cdf) given by

$$
\mathrm{G}(\mathrm{x} ; \alpha, \theta)=1-\theta^{\alpha}\left(\theta+\mathrm{x}^{2}\right)^{-\alpha}, \quad \mathrm{x}>0, \alpha, \theta>0
$$

where $\theta$ and $\alpha$ are the scale and shape parameters respectively. The probability density function (pdf) corresponding to Eq. (1) takes the form

$$
\mathrm{g}(\mathrm{x} ; \alpha, \theta)=2 \alpha \theta^{\alpha} \mathrm{x}\left(\theta+\mathrm{x}^{2}\right)^{-(\alpha+1)}, \quad \mathrm{x}>0, \alpha, \theta>0
$$

This study aims to suggest a new model namely the beta compound Rayleigh (BCR) distribution and studied some of its statistical properties. Moreover, the parameters of the new distribution are estimated by using the method of maximum likelihood. Two real data sets are used to show the effectiveness of the new distribution.
The rest of this paper is as follows. In Section 2, we define the beta compound Rayleigh (BCR) distribution and obtain some associated reliability functions. The limit of the BCR distribution is studied in Section 3. The expansion of BCR distribution is discussed in Section 4. In Section 5, some statistical properties of the new model are discussed. In Section 6, the maximum likelihood estimates and the observed information matrix are obtained. In Section 7, two applications of the new model are applied. Some concluding remarks have been given in the last Section.

\section{The BCR distribution}

In this section, we present the beta compound Rayleigh distribution and its sub-models. Some reliability functions associated to this distribution are also discussed.

Let $\mathrm{G}(\mathrm{x})$ be the cdf of any random variable $\mathrm{X}$. Eugene et al. [5] introduced a new procedure for building a new distribution from $\mathrm{G}(\mathrm{x})$ known as the beta generalized class of distribution given by

$F(x)=I_{G(x)}(a, b)=\frac{1}{\beta(a, b)} \int_{0}^{G(x)} u^{a-1}(1-u)^{b-1} d u$

where $\mathrm{a}>0$ and $\mathrm{b}>0$ are the additional shape parameters for the F distribution, $I_{y}(a, b)=\beta_{y}(a, b) / \beta(a, b)$ is the incomplete beta function ratio, $\beta_{y}(a, b)=\int_{0}^{y} u^{a-1}(1-u)^{b-1} d u$ is the incomplete beta function, $\beta(a, b)=\Gamma(a) \Gamma(b) / \Gamma(a+b)$ is the beta function and $\Gamma($.$) is$ the gamma function. The corresponding pdf for Eq. (3) is given by

$$
f(x)=\frac{1}{\beta(a, b)} g(x)[G(x)]^{a-1}[1-G(x)]^{b-1}
$$


where $\mathrm{g}(\mathrm{x})=\partial \mathrm{G}(\mathrm{x}) / \partial \mathrm{x}$ is the baseline density function. Replacing Eq. (1) in Eq. (3), we obtain a new distribution, so-called the beta compound Rayleigh (BCR) distribution with cdf given from

$\mathrm{F}(\mathrm{x} ; \alpha, \theta, \mathrm{a}, \mathrm{b})=\frac{1}{\beta(\mathrm{a}, \mathrm{b})} \int_{0}^{1-\theta^{\alpha}\left(\theta+\mathrm{x}^{2}\right)^{-\alpha}} \mathrm{u}^{\mathrm{a}-1}(1-\mathrm{u})^{\mathrm{b}-1} \mathrm{du}$

Or

$\mathrm{F}(\mathrm{x} ; \alpha, \theta, \mathrm{a}, \mathrm{b})=\mathrm{I}_{\left[1-\theta^{\alpha}\left(\theta+\mathrm{x}^{2}\right)^{-\alpha}\right]}(\mathrm{a}, \mathrm{b})=\frac{\beta\left[1-\theta^{\alpha}\left(\theta+\mathrm{x}^{2}\right)^{-\alpha}\right]^{(\mathrm{a}, \mathrm{b})}}{\beta(\mathrm{a}, \mathrm{b})}$

The pdf corresponding to Eq. (5) is given by

$f(x ; \alpha, \theta, a, b)=\frac{2 \alpha \theta^{\alpha b}}{\beta(a, b)} x\left(\theta+x^{2}\right)^{-(b \alpha+1)}\left[1-\theta^{\alpha}\left(\theta+x^{2}\right)^{-\alpha}\right]^{a-1}$

for $\mathrm{x}>0, \alpha>0, \theta>0, \mathrm{a}>0$ and $\mathrm{b}>0$.

For the survival analysis, the reliability function $\mathrm{R}(\mathrm{x})$, hazard function $h(x)$, inverse hazard function $h_{r}(x)$ and cumulative hazard function $\mathrm{H}(\mathrm{x})$ for the $\mathrm{BCR}$ distribution are givenrespectivelyas follows:

$$
\begin{aligned}
\mathrm{R}(\mathrm{x})=1-\mathrm{F}(\mathrm{x})= & 1-\frac{\beta_{\left[1-\theta^{\alpha}\left(\theta+\mathrm{x}^{2}\right)^{-\alpha}\right]}(\mathrm{a}, \mathrm{b})}{\beta(\mathrm{a}, \mathrm{b})}, \\
\mathrm{h}(\mathrm{x})=\frac{\mathrm{f}(\mathrm{x})}{\mathrm{R}(\mathrm{x})}= & 2 \alpha \theta^{\alpha \mathrm{b}} \mathrm{x}\left(\theta+\mathrm{x}^{2}\right)^{-(\mathrm{b} \alpha+1)} \\
& \times \frac{\left[1-\theta^{\alpha}\left(\theta+\mathrm{x}^{2}\right)^{-\alpha}\right]^{\mathrm{a}-1}}{\beta(\mathrm{a}, \mathrm{b})-\beta_{\left[1-\theta^{\alpha}\left(\theta+\mathrm{x}^{2}\right)^{-\alpha}\right]}(\mathrm{a}, \mathrm{b})} \\
\mathrm{h}_{\mathrm{r}}(\mathrm{x})=\frac{\mathrm{f}(\mathrm{x})}{\mathrm{F}(\mathrm{x})=} & 2 \alpha \theta^{\alpha \mathrm{b}} \mathrm{x}\left(\theta+\mathrm{x}^{2}\right)^{-(\mathrm{b} \alpha+1)} \\
& \times \frac{\left[1-\theta^{\alpha}\left(\theta+\mathrm{x}^{2}\right)^{-\alpha}\right]^{\mathrm{a}-1}}{\beta_{\left[1-\theta^{\alpha}\left(\theta+\mathrm{x}^{2}\right)^{-\alpha}\right]}(\mathrm{a}, \mathrm{b})}
\end{aligned}
$$

And

$$
H(x)=-\ln R(x)=-\ln \left[1-\frac{\beta_{\left[1-\theta^{\alpha}\left(\theta+x^{2}\right)^{-\alpha}\right]}(a, b)}{\beta(a, b)}\right]
$$

\subsection{Sub-models}

The following distributions can be obtained as special cases of the BCR distribution:

1) If $\mathrm{a}=1$, Eq. (6) reduces to the compound Rayleigh distribution, CR $(\alpha b, \theta)$.

2) When $\mathrm{a}=\mathrm{b}=1$, Eq. (6) represents the compound Rayleigh distribution, $\mathrm{CR}(\alpha, \theta)$.

3) Suppose $\theta=a=1$, then we obtain the compound Rayleigh distribution, CR $(\alpha b, 1)$.

4) Setting $\theta=a=b=1$, the $B C R$ distribution is reduced to the Burr-XII distribution, BXII ( $\alpha, 2)$.

5) Assume $\alpha=\theta=a=b=1$, Eq. (6) becomes the Burr-XII distribution, BXII ( 2,1$)$.

\section{The limit of the BCR distribution}

The limit of the beta compound Rayleigh distribution when $\mathrm{x} \rightarrow 0$ is 0 and when $\mathrm{x} \rightarrow \infty$ is 0 . We can show this by taking the limit of Eq. (6) as follows:

$$
\begin{aligned}
\lim _{x \rightarrow 0} f(x)= & \left(\lim _{x \rightarrow 0} \frac{2 \alpha \theta^{\alpha b}}{\beta(a, b)}\right)\left(\lim _{x \rightarrow 0} x\right) \times\left(\lim _{x \rightarrow 0}\left(\theta+x^{2}\right)^{-(b \alpha+1)}\right) \\
& \times\left(\lim _{x \rightarrow 0}\left[1-\theta^{\alpha}\left(\theta+x^{2}\right)^{-\alpha}\right]^{a-1}\right)=0
\end{aligned}
$$

Because $\lim _{x \rightarrow 0} x=0$ and $\lim _{x \rightarrow 0}\left[1-\theta^{\alpha}\left(\theta+x^{2}\right)^{-\alpha}\right]^{a-1}=0$.

Similarly, as $x \rightarrow \infty$, we can observe that by replacing the limit $\mathrm{x} \rightarrow 0$ with $\mathrm{x} \rightarrow \infty$, we get

$$
\lim _{x \rightarrow \infty} f(x)=0
$$

Because $\lim _{\mathrm{x} \rightarrow \infty}\left(\theta+\mathrm{x}^{2}\right)^{-(\mathrm{b} \alpha+1)}=0$

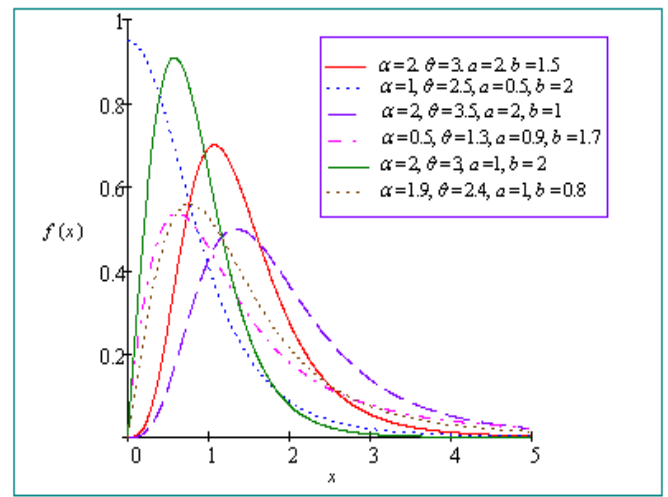

Fig. 1: The pdf of the BCR distribution for different values of the parameters.

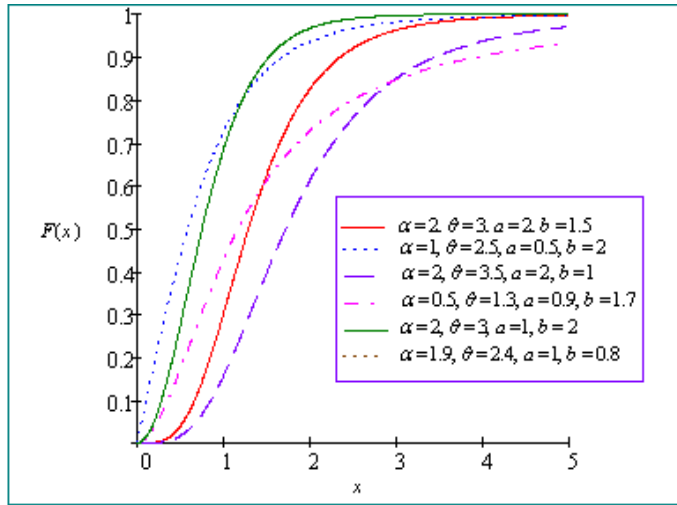

Fig. 2: The cdf of the BCR distribution for different values of the Parameters

\section{Expansion for the BCR distribution}

We can expand the cdf and pdf corresponding to the BCR distribution in terms of an infinite (or finite) weighted sums of cdf's and pdf's of random variables having CR distributions respectively. For $\mathrm{b}$ is a real non-integer,then we have the series representation 


$$
(1-u)^{b-1}=\sum_{j=0}^{\infty} \frac{(-1)^{j} \Gamma(b)}{j ! \Gamma(b-j)} u^{j}
$$

Therefore, the cdf of BCR distributionin Eq. (5) can be expressed as follows:

$$
F(x ; \alpha, \theta, a, b)=\sum_{j=0}^{\infty} z_{j} H^{a+j}(x ; \theta, \alpha)
$$

Where

$$
z_{j}=\frac{(-1)^{j} \Gamma(a+b)}{(a+j) j ! \Gamma(a) \Gamma(b-j)}
$$

and $\mathrm{H}(\mathrm{x} ; \theta, \alpha)$ deotes the cdf of $\mathrm{CR}$ distribution with parameters $\theta$ and $\alpha$. If $b$ is an integer, then the summation in Eq. (12) is stoped at $b-1$.

Likewise, we can express the pdf in Eq. (6) as below

$$
f(x ; \alpha, \theta, a, b)==\sum_{j=0}^{\infty} v_{j} D(x ; \theta, \alpha(b+j))
$$

Where

$$
v_{j}=\frac{(-1)^{j} \Gamma(a+b)}{(b+j) j ! \Gamma(b) \Gamma(a-j)}
$$

and $\mathrm{D}(\mathrm{x} ; \theta, \alpha(\mathrm{b}+\mathrm{j}))$ deotes the cdf of CR distribution with parameters $\theta$ and $\alpha(b+j)$. If $a$ is an integer, then the summation in Eq. (13) is stoped at $a-1$.

\section{Statistical properties}

In this section, we present a mathematical treatment of the proposed distribution such as; mean, variance, coefficient of variation, raw and incomplete moments, skewness, kurtosis, generating functions, Lorenz, Bonferroni and Zenga curves, Rényi of entropy, order statistics and record statistics.

\subsection{Raw moments}

Suppose $\mathrm{X}$ is a random variable distributed according to $\mathrm{BCR}$ distribtion, then the raw moments, say $\mu_{r}^{\prime}$ is given by

$$
\begin{aligned}
\mu_{\mathrm{r}}^{\prime}=\mathrm{E}\left(\mathrm{X}^{\mathrm{r}}\right) & =\frac{2 \alpha \theta^{\alpha \mathrm{b}}}{\beta(\mathrm{a}, \mathrm{b})} \int_{0}^{\infty} \mathrm{x}^{\mathrm{r}+1}\left(\theta+\mathrm{x}^{2}\right)^{-(\mathrm{b} \alpha+1)}\left[1-\theta^{\alpha}\left(\theta+\mathrm{x}^{2}\right)^{-\alpha}\right]^{\mathrm{a}-1} \mathrm{dx} \\
& =\frac{2 \alpha}{\theta \beta(\mathrm{a}, \mathrm{b})} \int_{0}^{\infty} \mathrm{x}^{\mathrm{r}+1}\left(1+\frac{\mathrm{x}^{2}}{\theta}\right)^{-(\mathrm{b} \alpha+1)}\left[1-\left(1+\frac{\mathrm{x}^{2}}{\theta}\right)^{-\alpha}\right]^{\mathrm{a}-1} \mathrm{dx}
\end{aligned}
$$

By using the binomial expansion in the last term of above integrand, we get

$$
\mu_{r}^{\prime}=\frac{2 \alpha}{\theta \beta(a, b)} \sum_{j=0}^{\infty} \frac{\left.(-1)^{j} \Gamma a\right)}{j ! \Gamma(a-j)} \int_{0}^{\infty} x^{r+1}\left(1+\frac{x^{2}}{\theta}\right)^{-[\alpha(b+j)+1]} d x
$$

Let $z=\left(1+\frac{x^{2}}{\theta}\right)^{-1}$ in the above equation, so we have

$$
\begin{aligned}
\mu_{r}^{\prime} & =\frac{\alpha \theta^{r / 2}}{\beta(a, b)} \sum_{j=0}^{\infty} \frac{\left.(-1)^{j} \Gamma a\right)}{j ! \Gamma(a-j)} \int_{0}^{1} z^{\alpha(b+j)-\frac{r}{2}-1}(1-z)^{r / 2} d z \\
& =\frac{\alpha \theta^{r / 2}}{\beta(a, b)} \sum_{j=0}^{\infty} \frac{\left.(-1)^{j} \Gamma a\right)}{j ! \Gamma(a-j)} \beta[\alpha(b+j)-r / 2, r / 2+1]
\end{aligned}
$$

Substituting $r=1,2$ in Eq. (14), then we get the mean and variance respectively as follows:

$$
\mu_{1}^{\prime}=\frac{\alpha \theta^{1 / 2}}{\beta(a, b)} \sum_{j=0}^{\infty} \frac{\left.(-1)^{j} \Gamma a\right)}{j ! \Gamma(a-j)} \beta[\alpha(b+j)-1 / 2,3 / 2]
$$

And

$$
v(x)=\frac{\alpha \theta}{\beta(a, b)}\left\{\begin{array}{l}
\sum_{j=0}^{\infty} \frac{\left.(-1)^{j} \Gamma a\right)}{j ! \Gamma(a-j)} \beta[\alpha(b+j)-1,2] \\
-\frac{\alpha}{\beta(a, b)}\left[\sum_{j=0}^{\infty} \frac{\left.(-1)^{j} \Gamma a\right)}{j ! \Gamma(a-j)} \beta[\alpha(b+j)-1 / 2,3 / 2]\right]^{2}
\end{array}\right\}
$$

\subsection{Coefficients of variation, skewness and kurtosis}

The coefficients of variation, skewness and kurtosis of the BCR distribution are given respectively as follows:

$$
\begin{aligned}
& \text { C.V }=\frac{\sigma}{\mu} \\
& =\frac{\beta^{1 / 2}(a, b) \sqrt{\left.-\frac{\alpha}{\beta(a, b)}\left[\sum_{j=0}^{\infty} \frac{\left.(-1)^{j} \Gamma a\right)}{j ! \Gamma(a-j)} \beta[\alpha(b+j)-1 / 2,3 / 2]\right]^{j} \Gamma a\right)} \beta[\alpha(b+j)-1,2]}{\alpha^{1 / 2} \sum_{j=0}^{\infty} \frac{\left.(-1)^{j} \Gamma a\right)}{j ! \Gamma(a-j)} \beta[\alpha(b+j)-1 / 2,3 / 2]}, \\
& \xi_{1}=\frac{\mu_{3}^{\prime}}{\left(\mu_{2}^{\prime}\right)^{3 / 2}}=\frac{\beta^{1 / 2}(a, b) \sum_{j=0}^{\infty} \frac{\left.(-1)^{j} \Gamma a\right)}{j ! \Gamma(a-j)} \beta[\alpha(b+j)-3 / 2,5 / 2]}{\alpha^{1 / 2}\left\{\sum_{j=0}^{\infty} \frac{\left.(-1)^{j} \Gamma a\right)}{j ! \Gamma(a-j)} \beta[\alpha(b+j)-1,2]\right\}^{3 / 2}}
\end{aligned}
$$

And

$$
\xi_{2}=\frac{\mu_{4}^{\prime}}{\left(\mu_{2}^{\prime}\right)^{2}}=\frac{\beta(a, b) \sum_{j=0}^{\infty} \frac{\left.(-1)^{j} \Gamma a\right)}{j ! \Gamma(a-j)} \beta[\alpha(b+j)-2,3]}{\alpha\left\{\sum_{j=0}^{\infty} \frac{\left.(-1)^{j} \Gamma a\right)}{j ! \Gamma(a-j)} \beta[\alpha(b+j)-1,2]\right\}^{2}}
$$

\subsection{Incomplete moments}

Suppose $\mathrm{X}$ is a random variable having the BCR distribution, then the $\mathrm{r}^{\text {th }}$ incomplete moments denoted as $\mathrm{m}_{\mathrm{r}}(\mathrm{z})$ can be obtained as follows:

$$
\begin{aligned}
\mathrm{m}_{\mathrm{r}}(\mathrm{z}) & =\int_{0}^{\mathrm{z}} \mathrm{x}^{\mathrm{r}} \mathrm{f}(\mathrm{x}) \mathrm{dx}=\frac{2 \alpha \theta^{\alpha b}}{\beta(a, b)} \\
& \quad \times \int_{0}^{\mathrm{z}} \mathrm{x}^{\mathrm{r}+1}\left(\theta+\mathrm{x}^{2}\right)^{-(b \alpha+1)}\left[1-\theta^{\alpha}\left(\theta+\mathrm{x}^{2}\right)^{-\alpha}\right]^{\mathrm{a}-1} \mathrm{dx}
\end{aligned}
$$


$m_{r}(z)=\frac{\alpha \theta^{r / 2}}{\beta(a, b)} \sum_{j=0}^{\infty} \frac{\left.(-1)^{j} \Gamma a\right)}{j ! \Gamma(a-j)} \int_{\left(1+\frac{z^{2}}{\theta}\right)^{-1}}^{1} y^{\alpha(b+j)-\frac{r}{2}-1}(1-y)^{r / 2} d y$

Based on the binomial expansion to the last factor, we get

$$
\begin{aligned}
m_{r}(z) & =\frac{\alpha \theta^{r / 2}}{\beta(a, b)} \sum_{j=0}^{\infty} \sum_{i=0}^{r / 2} \frac{(-1)^{i+j} \Gamma(a) \Gamma(r / 2+1)}{j ! i ! \Gamma(a-j) \Gamma(r / 2-i)} \int_{\left(1+\frac{z^{2}}{\theta}\right)^{-1}}^{1} y^{\alpha(b+j)+i-\frac{r}{2}-1} d y \\
& =\frac{\alpha \theta^{r / 2}}{\beta(a, b)} \sum_{j=0}^{\infty} \sum_{i=0}^{r / 2} \frac{(-1)^{i+j} \Gamma(a) \Gamma(r / 2+1)}{j ! i ! \Gamma(a-j) \Gamma(r / 2-i)} \\
& \times \frac{\left[1-\left(1+\frac{z^{2}}{\theta}\right)^{r / 2-[\alpha(b+j)+i]}\right]}{[\alpha(b+j)+i-r / 2]}
\end{aligned}
$$

\subsection{Moment and probability generating functions}

The moment generating function, say $\mathrm{M}_{\mathrm{x}}(\mathrm{t})$ of the BCR distribution can be obtained as follows:

$$
M_{x}(t)=E\left(e^{t x}\right)=\frac{2 \alpha \theta^{\alpha b}}{\beta(a, b)} \int_{0}^{\infty} e^{t x} x\left(\theta+x^{2}\right)^{-(b \alpha+1)}\left[1-\theta^{\alpha}\left(\theta+x^{2}\right)^{-\alpha}\right]^{a-1} d x
$$

Using $\mathrm{e}^{\mathrm{tx}}=\sum_{\mathrm{h}=0}^{\infty} \frac{\mathrm{t}^{\mathrm{h}} \mathrm{x}^{\mathrm{h}}}{\mathrm{h} !}$, then we obtain

$$
M_{x}(t)=\frac{\alpha}{\beta(a, b)} \sum_{h=0}^{\infty} \sum_{j=0}^{\infty} \frac{\left.t^{h} \theta^{h / 2}(-1)^{j} \Gamma a\right)}{h ! j ! \Gamma(a-j)} \beta[\alpha(b+j)-h / 2, h / 2+1]
$$

Similarly, the probability generating function denoted as $\mathrm{M}_{[\mathrm{x}]}(\mathrm{t})$ of the BCR distribution can be derived as below

$$
M_{[x]}(t)=E\left(t^{x}\right)=\frac{2 \alpha \theta^{\alpha b}}{\beta(a, b)} \int_{0}^{\infty} t^{x} x\left(\theta+x^{2}\right)^{-(b \alpha+1)}\left[1-\theta^{\alpha}\left(\theta+x^{2}\right)^{-\alpha}\right]^{a-1} d x
$$

Using $t^{\mathrm{x}}=\sum_{\mathrm{w}=0}^{\infty} \frac{(\ln \mathrm{t})^{\mathrm{w}} \mathrm{x}^{\mathrm{w}}}{\mathrm{w} !}$, then we have

$$
\begin{aligned}
M_{[x]}(t)= & \frac{\alpha}{\beta(a, b)} \sum_{w=0}^{\infty} \sum_{j=0}^{\infty} \frac{\left.(\ln t)^{w} \theta^{w / 2}(-1)^{j} \Gamma a\right)}{w ! j ! \Gamma(a-j)} \\
& \times \beta[\alpha(b+j)-w / 2, w / 2+1]
\end{aligned}
$$

\subsection{Lorenz, Bonferroni and Zenga curves}

The Lorenz, Bonferroni and Zenga curves have been used in different fields such as demography, insurance, reliability, medicine and economics (for more details see Kleiberand Kotz [6]). Oluyedeand Rajasooriya [7] defined the Lorenz $\mathrm{L}_{\mathrm{F}}(\mathrm{x})$, Bonferroni $\mathrm{B}(\mathrm{F}(\mathrm{x}))$ and Zenga $\mathrm{A}(\mathrm{x})$ curves respectively as follows:

$L_{F}(x)=\frac{1}{E(x)} \int_{0}^{x} t f(t) d t$,

$$
B(F(x))=\frac{1}{F(x) E(x)} \int_{0}^{x} t f(t) d t=\frac{L_{F}(x)}{F(x)}
$$

$\mathrm{A}(\mathrm{x})=1-\left[\frac{\mathrm{M}^{-}(\mathrm{x})}{\mathrm{M}^{+}(\mathrm{x})}\right]$

where $M^{-}(x)=\frac{1}{F(x)} \int_{0}^{x} t f(t) d t$ and $M^{+}(x)=\frac{1}{1-F(x)} \int_{x}^{\infty} t f(t) d t$

Therefore, these quantities for the BCR distribution are obtained below

$\mathrm{L}_{\mathrm{F}}(\mathrm{x})=\frac{\Omega_{1}}{\Omega_{2}}$

$\mathrm{B}(\mathrm{F}(\mathrm{x}))=\frac{\beta(\mathrm{a}, \mathrm{b}) \Omega_{1}}{\beta_{\left[1-\theta^{\alpha}\left(\theta+\mathrm{x}^{2}\right)^{-\alpha}\right]}(\mathrm{a}, \mathrm{b}) \Omega_{2}}$

And

$\mathrm{A}(\mathrm{x})=1-\frac{\Omega_{1} \Omega_{3}}{\Omega_{4}}$

Where

$$
\begin{aligned}
& \Omega_{1}=\sum_{\mathrm{h}=0}^{\infty} \sum_{\mathrm{j}=0}^{\infty}\left(\begin{array}{c}
\mathrm{a}-1 \\
\mathrm{j}
\end{array}\right)\left(\begin{array}{c}
1 / 2 \\
\mathrm{~h}
\end{array}\right) \frac{(-1)^{\mathrm{j}+\mathrm{h}}}{\left[\alpha(\mathrm{b}+\mathrm{j})+\mathrm{h}-\frac{1}{2}\right]}\left[1-\left(1+\frac{\mathrm{x}^{2}}{\theta}\right)^{-\left(\alpha(\mathrm{b}+\mathrm{j})+\mathrm{h}-\frac{1}{2}\right)}\right], \\
& \Omega_{2}=\sum_{\mathrm{j}=0}^{\infty}\left(\begin{array}{c}
\mathrm{a}-1 \\
\mathrm{j}
\end{array}\right)(-1)^{\mathrm{j}} \beta\left[\alpha(\mathrm{b}+\mathrm{j})-\frac{1}{2}, \frac{3}{2}\right] \\
& \Omega_{3}=\beta(\mathrm{a}, \mathrm{b})-\beta_{\left[1-\theta^{\alpha}\left(\theta+\mathrm{x}^{2}\right)^{-\alpha}\right]}(\mathrm{a}, \mathrm{b})
\end{aligned}
$$

And

$\Omega_{4}=\beta_{\left[1-\theta^{\alpha}\left(\theta+x^{2}\right)^{-\alpha}\right]}(a, b) \sum_{j=0}^{\infty}\left(\begin{array}{c}a-1 \\ j\end{array}\right)(-1)^{j} \beta_{\left[1+\frac{x^{2}}{\theta}\right]^{-1}}\left[\alpha(b+j)-\frac{1}{2}, \frac{3}{2}\right]$

\subsection{Rényi entropy}

The concept of entropy has been successfully used in different fields including statistic, queuing theory and reliability estimation. The entropy is a measure the variation of the uncertainty corresponding to the distribution of a random variable. The Rényi entropy is defined as

$\mathrm{I}_{\mathrm{R}}(\delta)=\frac{1}{1-\delta}[\log \mathrm{I}(\delta)]$

where $\mathrm{I}(\delta)=\int \mathrm{f}^{\delta}(\mathrm{x}) \mathrm{dx}, \delta>0$

$\mathrm{I}(\delta)=\int \mathrm{f}^{\delta}(\mathrm{x}) \mathrm{dx}, \delta>0$ and $\delta \neq 0$

Using Eq. (6) yields

$$
\begin{aligned}
& I(\delta)=\frac{2^{\delta} \alpha^{\delta} \theta^{\delta \alpha b}}{\beta^{\delta}(a, b)} \int_{0}^{\infty} x^{\delta}\left(\theta+x^{2}\right)^{-\delta(b \alpha+1)}\left[1-\theta^{\alpha}\left(\theta+x^{2}\right)^{-\alpha}\right]^{\delta(a-1)} d x \\
& =\frac{2^{\delta-1 / 2} \alpha^{\delta}}{\theta^{(\delta-1) / 2} \beta^{\delta}(a, b)} \sum_{j=0}^{\infty} \frac{(-1)^{j} \Gamma(\delta(a-1)+1)}{j ! \Gamma(\delta(a-1)-j+1)} \beta[\alpha(\delta b+j)+(\delta-1) / 2,(\delta+1) / 2]
\end{aligned}
$$

Therefore, the Rényi entropy is given below 


$$
\mathrm{I}_{\mathrm{R}}(\delta)=\frac{1}{1-\delta}\left\{\begin{array}{l}
\left(\delta-\frac{1}{2}\right) \log (2)+\delta \log (\alpha) \\
-\left(\frac{\delta-1}{2}\right) \log (\theta)-\delta \log [\beta(\mathrm{a}, \mathrm{b})] \\
+\sum_{\mathrm{j}=0}^{\infty} \log (\beta[\alpha(\delta \mathrm{b}+\mathrm{j})+(\delta-1) / 2,(\delta+1) / 2])
\end{array}\right\}
$$

\subsection{Order statistics}

Order statistics play an important role in probability and statistics. Let $\mathrm{x}_{1, \mathrm{n}} \leq \mathrm{x}_{2: \mathrm{n}}, \ldots \leq \mathrm{x}_{\mathrm{n}: \mathrm{n}}$ be the ordered sample from a continuous population with pdf $f(x)$ and $\operatorname{cdf} F(x)$. The pdf of $X_{k: n}$, the $k^{\text {th }}$ order statistics is given by

$$
\mathrm{f}_{\mathrm{X}_{\mathrm{kn}}}(\mathrm{x})=\frac{\mathrm{n} !}{(\mathrm{k}-1) !(\mathrm{n}-\mathrm{k}) !} \mathrm{f}(\mathrm{x})[\mathrm{F}(\mathrm{x})]^{\mathrm{k}-1}[1-\mathrm{F}(\mathrm{x})]^{\mathrm{n}-\mathrm{k}}, \mathrm{r}=1,2, \ldots, \mathrm{n}
$$

Then, the pdf the $\mathrm{k}^{\text {th }}$ order $\mathrm{BCR}$ random variable $\mathrm{X}_{\mathrm{k}: \mathrm{n}}$ can be obtained by using Eqs.(5) and (6) in above equation to be

$$
f_{X_{k n}}(x)=\frac{2 n ! \alpha \theta^{\alpha b}}{(k-1) !(n-k) !} I_{1} x\left(\theta+x^{2}\right)^{-(b \alpha+1)}\left[1-\theta^{\alpha}\left(\theta+x^{2}\right)^{-\alpha}\right]^{a-1}
$$

Where

$$
I_{1}=\sum_{j=0}^{n-k}\left(\begin{array}{c}
n-k \\
j
\end{array}\right)(-1)^{j} \frac{\beta_{\left[1-\theta^{\alpha}\left(\theta+x^{2}\right)^{-\alpha}\right]}^{k+j-b}(a, b)}{\beta^{k+j}(a, b)}
$$

Therefore, the pdf the $1^{\text {th }}$ order $\mathrm{BCR}$ random variable $\mathrm{X}_{1: \mathrm{n}}$ is given by

$$
\mathrm{f}_{\mathrm{X}_{\mathrm{ln}}}(\mathrm{x})=2 \mathrm{n} \alpha \theta^{\alpha \mathrm{b}} \mathrm{I}_{2} \mathrm{x}\left(\theta+\mathrm{x}^{2}\right)^{-(\mathrm{b} \alpha+1)}\left[1-\theta^{\alpha}\left(\theta+\mathrm{x}^{2}\right)^{-\alpha}\right]^{\mathrm{a}-1}
$$

Where

$$
I_{2}=\sum_{j=0}^{n-1}\left(\begin{array}{c}
n-1 \\
j
\end{array}\right)(-1)^{j} \frac{\beta_{\left[1-\theta^{\alpha}\left(\theta+x^{2}\right)^{-\alpha}\right]}^{j}(a, b)}{\beta^{j+1}(a, b)}
$$

Also, the pdf the $n^{\text {th }}$ order BCR random variable $X_{n: n}$ is given from

$$
f_{X_{n n}}(x)=2 n \alpha \theta^{\alpha b} I_{3} x\left(\theta+x^{2}\right)^{-(b \alpha+1)}\left[1-\theta^{\alpha}\left(\theta+x^{2}\right)^{-\alpha}\right]^{a-1}
$$

Where

$$
I_{3}=\beta^{-n}(a, b) \beta_{\left[1-\theta^{\alpha}\left(\theta+x^{2}\right)^{-\alpha}\right]}^{n-1}(a, b)
$$

Moreover, the joint distribution of two order statistics $X_{k: n} \leq X_{s: n}$ is given by

$$
\begin{aligned}
\mathrm{f}_{\mathrm{X}_{\mathrm{kss}}}\left(\mathrm{x}_{1}, \mathrm{x}_{2}\right)= & \frac{\mathrm{n} !}{(\mathrm{k}-1) !(\mathrm{s}-\mathrm{k}-1) !(\mathrm{n}-\mathrm{s}) !} \mathrm{f}\left(\mathrm{x}_{1}\right) \mathrm{f}\left(\mathrm{x}_{2}\right)\left[\mathrm{F}\left(\mathrm{x}_{1}\right)\right]^{\mathrm{k}-1} \\
& \times\left[\mathrm{F}\left(\mathrm{x}_{2}\right)-\mathrm{F}\left(\mathrm{x}_{1}\right)\right]^{\mathrm{s}-\mathrm{k}-1}\left[1-\mathrm{F}\left(\mathrm{x}_{2}\right)\right]^{\mathrm{n}-\mathrm{s}}
\end{aligned}
$$

Then, for the BCR distribution we obtain

$$
\mathrm{f}_{\mathrm{X}_{\mathrm{kss}}}\left(\mathrm{x}_{1}, \mathrm{x}_{2}\right)=\frac{4 \mathrm{n} ! \alpha^{2} \theta^{2 \alpha b}}{(\mathrm{k}-1) !(\mathrm{s}-\mathrm{k}-1) !(\mathrm{n}-\mathrm{s}) !} \mathrm{I}_{4} \mathrm{x}_{1} \mathrm{x}_{2}\left[\left(\theta+\mathrm{x}_{1}^{2}\right)\left(\theta+\mathrm{x}_{2}^{2}\right)\right]^{-(\mathrm{b} \alpha+1)}
$$

$$
\times\left[1-\theta^{\alpha}\left(\theta+x_{1}^{2}\right)^{-\alpha}\right]^{a-1}\left[1-\theta^{\alpha}\left(\theta+x_{2}^{2}\right)^{-\alpha}\right]^{a-1}
$$

Where

$$
\begin{aligned}
I_{4} & =\sum_{i=1}^{n-s s-k-1} \sum_{j=0}^{n}\left(\begin{array}{c}
n-s \\
i
\end{array}\right)\left(\begin{array}{c}
s-k-1 \\
j
\end{array}\right)(-1)^{i+j} \\
& \times \frac{\beta_{\left[1-\theta^{a}\left(\theta+x_{1}^{2}\right)^{-a}\right]}^{k+j-b}(a) \beta_{\left[1-\theta^{\alpha}\left(\theta+x_{2}^{2}\right)^{-a}\right]}^{s+i}(a, b)}{\beta^{s+i}(a, b)}
\end{aligned}
$$

\subsection{Record statistics}

Record values and the corresponding statistics are of interest and important in many real life applications including meteorological analysis, industrial stress testing, hydrology and athletic events. Let $X_{1}, X_{2}, \ldots, X_{n}$ be a sequence of independent and identically distributed (iid) random variables having $\operatorname{cdf} F(x)$ and $\operatorname{pdf} f(x)$. Set $Y_{n}=\max (\min )\left\{X_{1}, X_{2}, \ldots, X_{n}\right\}$ for $n \geq 1$. We call $X_{j}$ is an upper (or lower) record value of this sequence if $Y_{j}>(<) Y_{j-1}, j>1$. Thus $\mathrm{X}_{\mathrm{j}}$ will be named an upper (or lower) record value if its value is greater (or lower) than all previous observations.

The pdf of $X_{U(r)}$, the $r^{\text {th }}$ upper record is given as ( see Ahsanullah [8] and Arnold et al. [9])

$f_{X_{U(r)}}(x)=\frac{1}{(r-1) !}[R(x)]^{r-1} f(x)$

Where

$R(x)=-\ln [1-F(x)]$

Then, the pdf the $\mathrm{r}^{\text {th }}$ upper record BCR random variable $\mathrm{X}_{\mathrm{U}(\mathrm{r})}$ can be obtained to be

$\mathrm{f}_{\mathrm{X}_{\mathrm{U}(\mathrm{r})}}(\mathrm{x})=\frac{2 \alpha \theta^{\alpha \mathrm{b}}}{(\mathrm{r}-1) ! \beta(\mathrm{a}, \mathrm{b})} \mathrm{I}_{5}^{\mathrm{r}-1} \mathrm{x}\left(\theta+\mathrm{x}^{2}\right)^{-(\mathrm{b} \alpha+1)}\left[1-\theta^{\alpha}\left(\theta+\mathrm{x}^{2}\right)^{-\alpha}\right]^{\mathrm{a}-1}$

Where

$\mathrm{I}_{5}=\ln \left[\frac{\beta(\mathrm{a}, \mathrm{b})}{\beta(\mathrm{a}, \mathrm{b})-\beta_{\left[1-\theta^{\alpha}\left(\theta+\mathrm{x}_{2}^{2}\right)^{-\alpha}\right]}(\mathrm{a}, \mathrm{b})}\right]$

Furthermore, the joint distribution of the first $\mathrm{n}$ upper record values $\mathrm{x} \equiv\left(\mathrm{x}_{\mathrm{U}(1)}, \mathrm{x}_{\mathrm{U}(2)}, \ldots, \mathrm{x}_{\mathrm{U}(\mathrm{n})}\right)$ is given by (see Ahsanullah [8])

$\mathrm{f}_{1,2, \ldots, n}\left(\mathrm{x}_{\mathrm{U}(1)}, \mathrm{x}_{\mathrm{U}(2)}, \ldots, \mathrm{x}_{\mathrm{U}(\mathrm{n})}\right)=\mathrm{f}\left(\mathrm{x}_{\mathrm{U}(\mathrm{n})}\right) \prod_{\mathrm{i}=1}^{\mathrm{n}-1} \frac{\mathrm{f}\left(\mathrm{x}_{\mathrm{U}(\mathrm{i})}\right)}{1-\mathrm{F}\left(\mathrm{x}_{\mathrm{U}(\mathrm{i})}\right)}$

Consequently, for the BCR we get

$\mathrm{f}_{1,2, \ldots, \mathrm{n}}\left(\mathrm{x}_{\mathrm{U}(1)}, \mathrm{x}_{\mathrm{U}(2)}, \ldots, \mathrm{x}_{\mathrm{U}(\mathrm{n})}\right)=\frac{2^{\mathrm{n}} \alpha^{\mathrm{n}} \theta^{\mathrm{n} \alpha \mathrm{b}}}{\beta(\mathrm{a}, \mathrm{b})} \exp \left(\mathrm{I}_{6}+\mathrm{I}_{7}-\mathrm{I}_{8}\right)$

Where

$\mathrm{I}_{6}=\sum_{\mathrm{i}=1}^{\mathrm{n}} \ln \mathrm{x}_{\mathrm{i}}-(\mathrm{b} \alpha+1) \sum_{\mathrm{i}=1}^{\mathrm{n}} \ln \left(\theta+\mathrm{x}_{\mathrm{i}}^{2}\right)$, 


$$
\mathrm{I}_{7}=(\mathrm{a}-1) \sum_{\mathrm{i}=1}^{\mathrm{n}} \ln \left[1-\theta^{\alpha}\left(\theta+\mathrm{x}_{\mathrm{i}}^{2}\right)^{-\alpha}\right]
$$

And

$$
I_{8}=\sum_{i=1}^{n-1} \ln \left[\beta(a, b)-\beta_{\left[1-\theta^{\alpha}\left(\theta+x^{2}\right)^{-\alpha}\right]}(a, b)\right]
$$

Moreover, the pdf of $\mathrm{X}_{\mathrm{L}(\mathrm{r})}$, the $\mathrm{r}^{\text {th }}$ lower record is given as (see Ahsanullah [8] and Arnold et al. [9])

$$
f_{X_{U(r)}}(x)=\frac{1}{(r-1) !}[H(x)]^{r-1} f(x)
$$

Where

$$
H(x)=-\ln [F(x)]
$$

So, for the BCR distribution we have

$$
f_{X_{L(r)}}(x)=\frac{2 \alpha \theta^{\alpha b}}{(r-1) ! \beta(a, b)} I_{9}^{r-1} x\left(\theta+x^{2}\right)^{-(b \alpha+1)}\left[1-\theta^{\alpha}\left(\theta+x^{2}\right)^{-\alpha}\right]^{a-1}
$$

\section{Where}

$$
\mathrm{I}_{9}=\ln \beta(\mathrm{a}, \mathrm{b})-\ln \beta_{\left[1-\theta^{\alpha}\left(\theta+\mathrm{x}^{2}\right)^{-\alpha}\right]}(\mathrm{a}, \mathrm{b})
$$

In addition, the joint distribution of the first $\mathrm{n}$ lower record values $\mathrm{x} \equiv\left(\mathrm{x}_{\mathrm{L}(1)}, \mathrm{x}_{\mathrm{L}(2)}, \ldots, \mathrm{x}_{\mathrm{L}(\mathrm{n})}\right)$ is given by (see Ahsanullah [8])

$$
\mathrm{f}_{1,2, \ldots, \mathrm{n}}\left(\mathrm{x}_{\mathrm{L}(1)}, \mathrm{x}_{\mathrm{L}(2)}, \ldots, \mathrm{x}_{\mathrm{L}(\mathrm{n})}\right)=\mathrm{f}\left(\mathrm{x}_{\mathrm{L}(\mathrm{n})}\right) \prod_{\mathrm{i}=1}^{\mathrm{n}-1} \frac{\mathrm{f}\left(\mathrm{x}_{\mathrm{L}(\mathrm{i})}\right)}{\mathrm{F}\left(\mathrm{x}_{\mathrm{L}(\mathrm{i})}\right)}
$$

Then, for the new model we obtain

$$
\mathrm{f}_{1,2, \ldots, \mathrm{n}}\left(\mathrm{x}_{\mathrm{L}(1)}, \mathrm{x}_{\mathrm{L}(2)}, \ldots, \mathrm{x}_{\mathrm{L}(\mathrm{n})}\right)=2^{\mathrm{n}} \alpha^{\mathrm{n}} \theta^{\mathrm{n} \alpha \mathrm{b}} \exp \left(\mathrm{I}_{6}-\mathrm{I}_{7}-\mathrm{I}_{10}\right)
$$

Where

$$
I_{10}=\sum_{i=1}^{n-1} \ln \beta_{\left[1-\theta^{\alpha}\left(\theta+x^{2}\right)^{-a}\right]}(a, b)
$$

\section{Estimation of parameters}

In this section, we discuss the maximum likelihood estimates (MLEs) for the parameters of the BCR distribution. Let $\mathrm{x}_{1}, \mathrm{x}_{2}, \ldots, \mathrm{x}_{\mathrm{n}}$ be an independent random sample from the this distribution, then the corresponding log-likelihood function is given by

$$
\begin{aligned}
= & \mathrm{n} \ln (2)+\mathrm{n} \ln (\alpha)+\mathrm{n} \alpha \mathrm{b} \ln (\theta)-\mathrm{n} \ln (\Gamma(\mathrm{a}))-\mathrm{n} \ln (\Gamma(\mathrm{b}))+\mathrm{n} \ln (\Gamma(\mathrm{a}+\mathrm{b})) \\
& +\sum_{\mathrm{i}=1}^{\mathrm{n}} \ln \left(\mathrm{x}_{\mathrm{i}}\right)-(\mathrm{b} \alpha+1) \sum_{\mathrm{i}=1}^{\mathrm{n}} \ln \left(\mathrm{m}_{\mathrm{i}}\right)+(\mathrm{a}-1) \sum_{\mathrm{i}=1}^{\mathrm{n}} \ln \left(\mathrm{w}_{\mathrm{i}}\right)
\end{aligned}
$$

Where $\mathrm{m}_{\mathrm{i}}=\theta+\mathrm{x}_{\mathrm{i}}^{2}$ and $\mathrm{w}_{\mathrm{i}}=1-\theta^{\alpha} \mathrm{m}_{\mathrm{i}}^{-\alpha}$

The components of the score vector $\nabla=\left(\frac{\partial}{\partial \alpha}, \frac{\partial}{\partial \theta}, \frac{\partial}{\partial \mathrm{a}}, \frac{\partial}{\partial \mathrm{b}}\right)$ are given below:

$$
\frac{\partial}{\partial \alpha}=\mathrm{n}\left[\frac{1}{\alpha}+\mathrm{b} \ln (\theta)\right]-\mathrm{b} \sum_{\mathrm{i}=1}^{\mathrm{n}} \ln \left(\mathrm{m}_{\mathrm{i}}\right)-(\mathrm{a}-1) \theta^{\alpha} \sum_{\mathrm{i}=1}^{\mathrm{n}}\left[\frac{\ln (\theta)-\ln \left(\mathrm{m}_{\mathrm{i}}\right)}{\mathrm{m}_{\mathrm{i}}^{\alpha} \mathrm{w}_{\mathrm{i}}}\right],
$$

$\frac{\partial}{\partial \theta}=\frac{n \alpha b}{\theta}-(\alpha b+1) \sum_{i=1}^{n} m_{i}^{-1}-\alpha(a-1) \theta^{\alpha-1} \sum_{i=1}^{n}\left[\frac{x_{i}^{2}}{m_{i}^{\alpha+1} w_{i}}\right]$,

$\frac{\partial}{\partial \mathrm{a}}=-\mathrm{n}\left[\Psi^{(0)}(\mathrm{a})-\Psi^{(0)}(\mathrm{a}+\mathrm{b})\right]+\sum_{\mathrm{i}=1}^{\mathrm{n}} \ln \left(\mathrm{w}_{\mathrm{i}}\right)$

And

$\frac{\partial}{\partial \mathrm{b}}=\mathrm{n}\left\{\alpha \ln (\theta)-\left[\Psi^{(0)}(\mathrm{b})-\Psi^{(0)}(\mathrm{a}+\mathrm{b})\right]\right\}-\alpha \sum_{\mathrm{i}=1}^{\mathrm{n}} \ln \left(\mathrm{m}_{\mathrm{i}}\right)$

where $\Psi^{(\mathrm{n})}(\mathrm{x})$ is the polygamma function. The MLEs, say $(\hat{\alpha}, \hat{\theta}, \hat{a}, \hat{b})$ of $(\alpha, \theta, a, b)$ can be obtained by solving the system of nonlinear equations (35) through (38). These equations cannot be solved analytically and it needed iterative techniques such as Newton-Raphson algorithm.

For the purposes of interval estimation and testing of hypotheses for the model parameters $(\alpha, \theta, a, b)$, we derive the $4 \times 4$ observed information matrix $\mathrm{J}(\Theta)=\left\{\mathrm{J}_{\mathrm{wv}}\right\} \quad($ For $\mathrm{w}, \mathrm{v}=\alpha, \theta, \mathrm{a}, \mathrm{b})$

To be

$\mathrm{J}(\Theta)=\left[\begin{array}{cccc}\mathrm{J}_{\alpha \alpha} & \mathrm{J}_{\alpha \theta} & \mathrm{J}_{\alpha \mathrm{a}} & \mathrm{J}_{\alpha \mathrm{b}} \\ \mathrm{J}_{\theta \alpha} & \mathrm{J}_{\theta \theta} & \mathrm{J}_{\theta \mathrm{a}} & \mathrm{J}_{\theta \mathrm{b}} \\ \mathrm{J}_{\mathrm{a} \alpha} & \mathrm{J}_{\mathrm{a} \theta} & \mathrm{J}_{\mathrm{aa}} & \mathrm{J}_{\mathrm{ab}} \\ \mathrm{J}_{\mathrm{b} \alpha} & \mathrm{J}_{\mathrm{b} \theta} & \mathrm{J}_{\mathrm{ba}} & \mathrm{J}_{\mathrm{bb}}\end{array}\right]$

Whose elements are obtained in Appendix A.

\section{Applications}

In this section, we present two applications of the BCR distribution to two real data sets. The first data set consists of 63 observations of the strengths of $1.5 \mathrm{~cm}$ glass fibers which obtained by workers at the UK National Physical Laboratory. The data are: $0.55,0.74,0.77,0.81,0.84,0.93,1.04,1.11,1.13,1.24,1.25,1.27$, $1.28,1.29,1.30,1.36,1.39,1.42,1.48,1.48,1.49,1.49,1.50,1.50$, $1.51,1.52,1.53,1.54,1.55,1.55,1.58,1.59,1.60,1.61,1.61,1.61$, $1.61,1.62,1.62,1.63,1.64,1.66,1.66,1.66,1.67,1.68,1.68,1.69$, $1.70,1.70,1.73,1.76,1.76,1.77,1.78,1.81,1.82,1.84,1.84,1.89$, $2.00,2.01,2.24$. These data have also been used by Smith \& Naylor [10] and Merovci et al. [11].The second data set represents the relief times of 20 patients receiving an analgesic which are given by Gross and Clark [12]. The data are: 1.1, 1.4, 1.3, 1.7, 1.9, $1.8,1.6,2.2,1.7,2.7,4.1,1.8,1.5,1.2,1.4,3,1.7,2.3,1.6$, 2.These data set is previously studied by Rodrigues et al. [13] and Mead [14].

We use these two data sets to compare the fit of the new model, beta compound Rayleigh (BCR) with six models: compound Rayleigh (CR), Rayleigh (R), Burr-XII (BXII), generalized Rayleigh (GR), transmuted generalized Rayleigh (TGR) (Morovci[15]) and exponentiated transmuted generalized Rayleigh (ETGR) (Afify et al. [16]).

First, we derive the maximum likelihood estimates (MLEs) for each model and then comparing the results withinsome goodnessof-fit statistics AIC (Akaike information criterion), AICC (corrected Akaike information criterion), CAIC (consistent Akaike information criterion) and BIC (Bayesian information criterion). The better model corresponds to smaller AIC, AICC, CAIC and BICvalues.

Where

$\mathrm{AIC}=2 \mathrm{k}-2 \hat{(.),} \quad \mathrm{AICC}=\mathrm{AIC}+\frac{2 \mathrm{k}(\mathrm{k}+1)}{\mathrm{n}-\mathrm{k}-1}$ 
$\mathrm{CAIC}=\frac{2 \mathrm{kn}}{\mathrm{n}-\mathrm{k}-1}-2 \hat{(.),} \mathrm{BIC}=\mathrm{k} \log (\mathrm{n})-2 \hat{(.)}$ Where $\hat{(.)}$

and the values of AIC, AICC, CAIC and BIC displayed in Tables $(1-2)$.

Denotes the log-likelihood function evaluated at the MLEs, $\mathrm{k}$ is the number of parameters, and $\mathrm{n}$ is the sample size. The MLEs

Table 1: MLEs for BCR, CR, R, BXII, GR, TGR and ETGR Models and the Statistics AIC, AICC, CAIC And BIC; First Data Set

\begin{tabular}{|c|c|c|c|c|c|c|c|c|c|}
\hline \multirow{2}{*}{ Model } & \multicolumn{4}{|l|}{ Estimates } & \multicolumn{5}{|l|}{ Statistics } \\
\hline & $\hat{\alpha}$ & $\hat{\theta}$ & $\hat{a}$ & $\hat{b}$ & $-2 \hat{}$ & AIC & AICC & CAIC & BIC \\
\hline BCR & 21.907 & 332.532 & 5.067 & 30.558 & 41.848 & 49.848 & 50.538 & 50.538 & 58.421 \\
\hline $\mathrm{CR}$ & $5.228 \times 10^{3}$ & $1.241 \times 10^{4}$ & -- & -- & 99.592 & 103.592 & 103.792 & 103.792 & 107.878 \\
\hline $\mathrm{R}$ & -- & 1.187 & -- & -- & 159.841 & 161.841 & 161.906 & 161.906 & 163.984 \\
\hline BXII & 0.321 & 7.482 & -- & -- & 97.442 & 101.442 & 101.642 & 101.642 & 105.729 \\
\hline GR & 5.486 & 0.987 & -- & -- & 47.858 & 51.858 & 52.058 & 52.058 & 56.144 \\
\hline TGR & 5.372 & 0.932 & 0.312 & -- & 49.319 & 55.319 & 55.725 & 55.725 & 61.748 \\
\hline
\end{tabular}

Table 2: MLEs for BCR, CR, R, BXII, GR, TGR and ETGR Models and the Statistics AIC, AICC, CAIC and BIC; Second Data Set

\begin{tabular}{|c|c|c|c|c|c|c|c|c|c|}
\hline \multirow[b]{2}{*}{ Model } & \multicolumn{4}{|l|}{ Estimates } & \multicolumn{5}{|l|}{ tatistics } \\
\hline & $\hat{\alpha}$ & $\hat{\theta}$ & $\hat{a}$ & $\hat{b}$ & $-2 \hat{}$ & AIC & AICC & CAIC & BIC \\
\hline BCR & 5.841 & 1.699 & 58.076 & 0.421 & 30.81 & 38.81 & 41.477 & 41.477 & 42.793 \\
\hline $\mathrm{CR}$ & 201.728 & 819.593 & -- & -- & 44.985 & 48.985 & 49.691 & 49.691 & 50.977 \\
\hline $\mathrm{R}$ & -- & 2.041 & -- & -- & 85.76 & 87.76 & 87.982 & 87.982 & 88.755 \\
\hline BXII & 0.018 & 95.12 & -- & -- & 42.415 & 46.415 & 47.12 & 47.12 & 48.406 \\
\hline GR & 3.246 & 0.691 & -- & -- & 36.805 & 40.805 & 41.51 & 41.51 & 42.796 \\
\hline TGR & 3.329 & 0.635 & 0.592 & -- & 35.424 & 41.424 & 42.924 & 42.924 & 44.412 \\
\hline
\end{tabular}

From Tables 1 and 2, we can notice that the BCR model has the smallest values for the AIC, AICC, CAIC and BIC statistics between all fitted distributions. Consequently, we can deduct that the BCR distribution can provide a significantly better fit than the other models.

\section{Conclusion}

This paper introduces a new distribution namely the beta compound Rayleigh (BCR) distribution which generalizes the compound Rayleigh (CR) distribution. Important properties of the new distribution are studied including the mean, variance, coefficient of variation, raw and incomplete moments, skewness, kurtosis, moment and probability generating functions, reliability, hazard, reverse hazard and cumulative hazard functions, Lorenz, Bonferroni and Zenga curves, Rényi of entropy, order statistics and record statistics. The parameters of the new distribution are estimated by using the maximum likelihood criteria and the observed Fisher information matrix is derived. Two real data sets are used to show that the new model can present a better fit than some other known distributions.

\section{References}

[1] T.A. Abushal, Estimation of the unknown parameters for the compound Rayleigh distribution based on progressive first-failurecensored sampling, Open Journal of Statistics, 1 (2011) 161-171. https://doi.org/10.4236/ojs.2011.13020.

[2] O. Shajaee, R. Azimi and M. Babanezhad, Empirical Bayes estimators of parameter and reliability function for compound Rayleigh distribution under record data,American Journal of Theoretical andApplied Statistics, 1 (2012) 12-15.

[3] D.R. Barot, and M.N. Patal, Performance of estimates of reliability parameters for compound Rayleigh progressive type-ii censored data, Control Theory and Informatics, 5 (2015) 33-41.

[4] G.A. Abd-Elmougod, and E.E. Mahmoud, Parameters estimation of compound Ratleigh distribution under an adaptive type-ii progressive hybrid censored data for constant partially accelerated life tests. Global Journal of Pure and Applied Mathematics, 12(2016) 3253-3273.
[5] N. Eugene, C. Lee, F. Famoye, The beta-normal distribution and its applications,Communication in Statistics-Theory and Methods, 31(2002) 497-512. https://doi.org/10.1081/STA-120003130.

[6] C. Kleiber, S.Kotz, Statistical Size Distributions in Economics and Actuarial Sciences, Wiley Series in Probability and Statistics. John Wily \& Sons, 2003

[7] B.O. Oluyede, S. Rajasooriya, The Mc-Dagum distribution and its statistical properties with applications,Asian Journal of Mathematics and Applications, 44(2013) 1-16.

[8] M. Ahsanullah,Record Statistics. New York: Nova Science Publishers, Inc. Commack, 1995.

[9] B.C. Arnold, N. Balakrishan, H.N. Nagaraja,Records. New York, Wiley, USA, 1998.

[10] R.L. Smith, J.C. Naylor, A comparison of maximum likelihood estimators for the three-parameter Weibull distribution, Appl Stat, 36(1987) 358:369.

[11] F. Merovci, M.A.Khaleel, N.A., Ibrahim, M. Shitan, The beta Burr type $\mathrm{x}$ distribution properties with applications, Springer Plus, 5(2016):697.doi: 10.1186/s40064-016-2271-9. eCollection 2016

[12] A.J. Gross, V. A. Clark, Survival distributions: Reliability applications in the binomial sciences, John Wiley and Sons, New York, 1975

[13] J.A. Rodrigues, A.P.C. Silva, G.G. Hamedani, The beta exponential Lindley distribution, Journalof Statistical Theory and Applications, 14(2014):60-75. https://doi.org/10.2991/jsta.2015.14.1.6.

[14] M.E. Mead, On five- parameter Lomax distribution: properties and applications,PakistanJournal of Statistics and Operations Research, 1(2016) 185-199.

[15] F. Merovci, Transmuted generalized Rayleigh distribution, AJ. Stat. Appl, 3, 1(2014):9-20.

[16] A.Z. Afify, ZM. Nofal, A.E. Ebraheim, Exponential transmuted generalized Rayleigh distribution: A new four parameter Rayleigh distribution, Pakistan Journal of Statistics and Operations Research, XI,1(2015) 115-134. https://doi.org/10.18187/pjsor.v11i1.873.

\section{Appendix A}

The elements of the observed information matrix are given below

$\mathrm{J}_{\alpha \alpha}=\frac{-\mathrm{n}}{\alpha^{2}}-(\mathrm{a}-1) \theta^{\alpha} \sum_{\mathrm{i}=1}^{\mathrm{n}}\left[\frac{\left(\ln (\theta)-\ln \left(\mathrm{m}_{\mathrm{i}}\right)\right)^{2}}{\mathrm{~m}_{\mathrm{i}}^{\alpha} \mathrm{w}_{\mathrm{i}}^{2}}\right]$, 


$$
\begin{aligned}
& \mathrm{J}_{\alpha \theta}=\frac{\mathrm{nb}}{\theta}-\mathrm{b} \sum_{\mathrm{i}=1}^{\mathrm{n}} \mathrm{m}_{\mathrm{i}}^{-1}-(\mathrm{a}-1) \theta^{\alpha-1} \sum_{\mathrm{i}=1}^{\mathrm{n}}\left\{\frac{\mathrm{x}_{\mathrm{i}}^{2}\left[\mathrm{w}_{\mathrm{i}}+\alpha\left(\ln (\theta)-\ln \left(\mathrm{m}_{\mathrm{i}}\right)\right)\right]}{\mathrm{m}_{\mathrm{i}}^{\alpha+1} \mathrm{w}_{\mathrm{i}}^{2}}\right\}, \\
& J_{\alpha a}=-\theta^{\alpha} \sum_{i=1}^{n}\left[\frac{\ln (\theta)-\ln \left(m_{i}\right)}{m_{i}^{\alpha} w_{i}}\right] \\
& \mathrm{J}_{\alpha \mathrm{b}}=\mathrm{n} \ln (\theta)-\sum_{\mathrm{i}=1}^{\mathrm{n}} \ln \left(\mathrm{m}_{\mathrm{i}}\right), \\
& \mathrm{J}_{\theta \theta}=\frac{-\mathrm{n} \alpha \mathrm{b}}{\theta^{2}}+(\alpha \mathrm{b}+1) \sum_{\mathrm{i}=1}^{\mathrm{n}} \mathrm{m}_{\mathrm{i}}^{-2} \\
& -\alpha(a-1) \theta^{\alpha-2} \sum_{i=1}^{n}\left\{\frac{x_{i}^{2}\left[m_{i}^{\alpha} w_{i}\left((\alpha-1) x_{i}^{2}-2 \theta\right)+\alpha \theta^{\alpha} x_{i}^{2}\right]}{m_{i}^{2(\alpha+1)} w_{i}^{2}}\right\}, \\
& \mathrm{J}_{\theta \mathrm{a}}=-\alpha \theta^{\alpha-1} \sum_{\mathrm{i}=1}^{\mathrm{n}}\left[\frac{\mathrm{x}_{\mathrm{i}}^{2}}{\mathrm{~m}_{\mathrm{i}}^{\alpha+1} \mathrm{w}_{\mathrm{i}}}\right], \\
& \mathrm{J}_{\theta b}=\frac{\mathrm{n} \alpha}{\theta}-\alpha \sum_{\mathrm{i}=1}^{\mathrm{n}} \mathrm{m}_{\mathrm{i}}^{-1}, \\
& \mathrm{~J}_{\mathrm{aa}}=-\mathrm{n}\left\{\left(\Psi^{(0)}(\mathrm{a})-\Psi^{(0)}(\mathrm{a}+\mathrm{b})\right)^{2}+\Psi^{(1)}(\mathrm{a})-\Psi^{(1)}(\mathrm{a}+\mathrm{b})\right\}, \\
& J_{a b}=-n\left\{\left(\Psi^{(0)}(a)-\Psi^{(0)}(a+b)\right)\left(\Psi^{(0)}(b)-\Psi^{(0)}(a+b)\right)-\Psi^{(1)}(a+b)\right\}
\end{aligned}
$$

And

$\mathrm{J}_{\mathrm{bb}}=-\mathrm{n}\left\{\left(\Psi^{(0)}(\mathrm{b})-\Psi^{(0)}(\mathrm{a}+\mathrm{b})\right)^{2}+\Psi^{(1)}(\mathrm{b})-\Psi^{(1)}(\mathrm{a}+\mathrm{b})\right\}$. 